\title{
Recapping The Latest Vaccine Developments
}

\author{
By: Atikaran Krishnamoorthy (D)
}

Since the first case was identified in Wuhan, China in November 2019, there have been 84.5 million cases of COVID-19 and 1.8 million deaths from this virus globally as of January $5,2021^{1}$. To combat this virus' spread, various strategies have been employed, such as mask mandates, social distancing, increased COVID-19 testing, and contact tracing. Since December 2020, another strategy has been made available: vaccines. This is because three vaccines that showed promising Phase 3 results are currently in use to help curb COVID-19.

On November 9, Pfizer and BioNTech both announced that the mRNA vaccine that they collaborated on (BNT162b2) was found to be $90 \%$ effective based on an interim analysis of their phase 3 study results ${ }^{2}$. A phase 3 trial consists of a diverse group of thousands of participants and each participant is either given the vaccine or a placebo ${ }^{3}$. The purpose of a phase 3 study is to understand how effective the vaccine is and to learn more about how safe it is ${ }^{3}$. Pfizer's final phase 3 results, released on November 18, showed that their vaccine's efficacy was in fact $95 \%{ }^{4}$, which is better than what their interim analysis had shown.

In addition to the Pfizer-BioNTech vaccine, Moderna announced on November 16 that an interim analysis of their phase 3 results found that their mRNA vaccine (mRNA-1273) was $94.5 \%$ effective ${ }^{5}$. The final efficacy rate for Moderna's vaccine was $94.1 \%$ based on the final analysis released on November $30^{6}$.

As previously mentioned, Pfizer's and Moderna's vaccines are both mRNA vaccines. This means that they consist of mRNA, which is then translated in our cells, thus giving rise to COVID-19 S or Spike protein ${ }^{5,7}$. After, this allows for the development of adaptive immunity against COVID-19. Unlike Pfizer's and Moderna's vaccines, AstraZeneca has developed a vaccine that is not a mRNA vaccine. Instead, it is a weakened form of the common cold (adenovirus) that normally infects chimpanzees and it consists of DNA that codes for the COVID-19 Spike protein ${ }^{8}$.
On November 23, AstraZeneca published a press release stating that their vaccine (AZD1222) had an average efficacy of $70 \%{ }^{9}$. The reason for the mediocre efficacy was that a dosing error had occurred ${ }^{10}$. Instead of receiving one full dose followed by another full dose at least a month later, 2741 out of 11636 participants initially received a half dose followed by a full one $e^{9,10}$. However, the mistake may have been a blessing in disguise. The reason-the latter dosing regimen was found to be $90 \%$ effective versus the original regimen, which was only $62 \%$ effective $^{9}$.

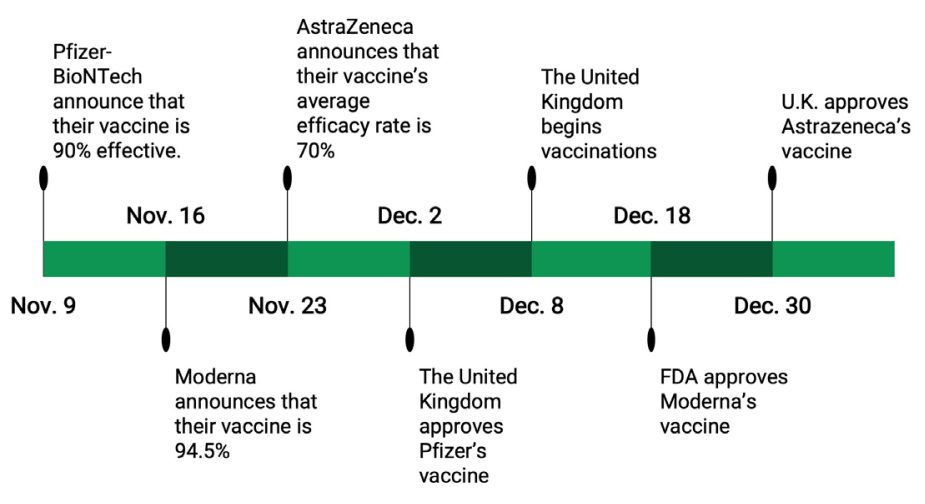

With these great developments begins the race for drug agencies around the world to approve the vaccines. The United Kingdom approved Pfizer's vaccine for emergency use on December $2^{11}$. Canada did the same on December $9^{12}$, while the United States did so on December $11^{13}$. On Dec. 18, Moderna's vaccine was approved for emergency use by the U.S. ${ }^{14}$, while Canada approved it on December 2315. On December 30 , the U.K. gave AstraZeneca's vaccine the green light $^{16}$. In all three nations, vaccinations are currently underway ${ }^{17,18,19}$.

With the progress on vaccines, there are also concerns and doubts regarding them. For example, one concern is that the trials of Pfizer and Moderna did not include pregnant women, so it is unclear how these vaccines affect pregnant women ${ }^{20}$. In addition, some participants in the Pfizer and Moderna vaccine trials experienced 
side effects, such as headaches and fever, and there is fear that this could dissuade patients from receiving a second dose, which all three vaccines require ${ }^{21}$. Furthermore, while the Moderna vaccine could be stored at around $-20^{\circ} \mathrm{C}$ (which standard medical freezers can generate $)^{22}$ and the AstraZeneca vaccine could be stored at normal refrigerator temperatures $\left(2-8^{\circ} \mathrm{C}\right)^{9}$, the Pfizer vaccine must be stored at temperatures of about $-70^{\circ} \mathrm{C}^{22}$. This would require heavy duty freezers that are not readily available in developing nations and rural areas $^{18}$.

Despite concerns such as these, the recent vaccine developments allow for great optimism regarding what lies ahead. With speedy approvals from drug regulatory agencies around the globe and quick deployment, these vaccines could hopefully put an end to this pandemic that has claimed the lives of many people. To add, the race to develop a vaccine against COVID-19 reminds us that when people come together, there is nothing that we cannot do.

Atikaran Krishnamoorthy is a Staff Writer for the JULS. Please address correspondence to: atikaran.krishnamoorthy@mail.utoronto.ca

\section{COMPETING INTERESTS}

No competing interests declared.

Received: December 18, 2020

Accepted: January 7, 2021

Published online: March 22, 2021

\section{REFERENCES}

1. WHO Coronavirus Disease (COVID-19) Dashboard. World Health Organization https://covid19.who.int/ (2020).

2. Pfizer and BioNTech Announce Vaccine Candidate Against COVID-19 Achieved Success In First Interim Analysis From Phase 3 Study. Pfizer https://www.pfizer.com/news/press-release/pressrelease-detail/pfizer-and-biontech-announce-vaccine-candidateagainst (2020).

3. Emergency Use Authorization for Vaccines Explained. U.S. Food \& Drug Administration https://www.fda.gov/vaccines-bloodbiologics/vaccines/emergency-use-authorization-vaccinesexplained (2020).

4. Pfizer and BioNTech Conclude Phase 3 Study of COVID-19 Vaccine Candidate, Meeting All Primary Efficacy Endpoints. Pfizer https://www.pfizer.com/news/press-release/press-release-detail/ pfizer-and-biontech-conclude-phase-3-study-covid-19-vaccine (2020).

5. Moderna's COVID-19 Vaccine Candidate Meets its Primary Efficacy Endpoint in the First Interim Analysis of the Phase 3 COVE Study. Moderna https://investors.modernatx.com/news-releases/ news-release-details/modernas-covid-19-vaccine-candidatemeets-its-primary-efficacy (2020).

6. Moderna Announces Primary Efficacy Analysis in Phase 3
COVE Study for Its COVID-19 Vaccine Candidate and Filing Today with U.S. FDA for Emergency Use Authorization. Moderna https:// investors.modernatx.com/news-releases/news-release-details/ moderna-announces-primary-efficacy-analysis-phase-3-covestudy (2020).

7. BioNTech and Pfizer Initiate Rolling Submission To European Medicines Agency For SARS-COV-2 Vaccine Candidate BNT162b2.

Pfizer https://www.pfizer.com/news/press-release/press-releasedetail/biontech-and-pfizer-initiate-rolling-submission-european (2020).

8. Corum, J. \& Zimmer, C. How the Oxford-AstraZeneca Vaccine Works. The New York Times https://www.nytimes.com/ interactive/2020/health/oxford-astrazeneca-covid-19-vaccine.html (2020).

9. AZD1222 vaccine met primary efficacy endpoint in preventing COVID-19. AstraZeneca https://www.astrazeneca.com/content/ astraz/media-centre/press-releases/2020/azd1222hlr.html (2020).

10. Murray, J. Oxford Covid vaccine hit $90 \%$ success rate thanks to dosing error. The Guardian https://www.theguardian.com/ uk-news/2020/nov/23/oxford-covid-vaccine-hit-90-success-ratethanks-to-dosing-error?CMP=Share_iOSApp_Other (2020).

11. Boseley, S. \& Halliday, J. UK approves Pfizer/BioNTech Covid vaccine for rollout next week. The Guardian https://www. theguardian.com/society/2020/dec/02/pfizer-biontech-covidvaccine-wins-licence-for-use-in-the-uk (2020).

12. Cecco, L. Canada approves Pfizer vaccine and plans to begin rollout next week as US awaits decision. The Guardian https:// www.theguardian.com/world/2020/dec/09/canada-approvespfizer-vaccine-and-plans-to-begin-rollout-next-week-as-us-awaitsdecision (2020).

13. Glenza, J. FDA approves Pfizer/BioNTech coronavirus vaccine for emergency use in US. The Guardian https://www.theguardian. com/world/2020/dec/11/fda-approves-pfizer-biontech-covid-19coronavirus-vaccine-for-use-in-us (2020).

14. FDA Takes Additional Action in Fight Against COVID-19 By Issuing Emergency Use Authorization for Second COVID-19 Vaccine. U.S. Food \& Drug Administration https://www.fda.gov/ news-events/press-announcements/fda-takes-additional-actionfight-against-covid-19-issuing-emergency-use-authorizationsecond-covid (2020).

15. Dangerfield, K. Canada approves Moderna coronavirus vaccine, 1st doses to arrive in 'coming days'. Global News https:// globalnews.ca/news/7539137/moderna-coronavirus-vaccinecanada-approved/ (2020).

16. Talmazan, Y. AstraZeneca's Covid-19 vaccine approved in the U.K. NBC News https://www.nbcnews.com/news/world/ astrazeneca-s-covid-19-vaccine-approved-u-k-n1252418 (2020).

17. Diaz, J. U.K. Begins Nationwide Coronavirus Immunization, Largest In Nation's History. National Public Radio https://www.npr. org/sections/coronavirus-live-updates/2020/12/08/944125280/uk-begins-nationwide-coronavirus-immunization-largest-in-nationshistory (2020).

18. Jacobs, E. Canada Administers Its 1st COVID-19 Vaccine Shots. National Public Radio https://www.npr.org/sections/coronaviruslive-updates/2020/12/14/946301025/canada-administers-its-1stcovid-19-vaccine-shots (2020).

19. West, M. G. New York City Kicks Off Covid-19 Vaccine Drive. The Wall Street Journal https://www.wsj.com/articles/ 
queens-nurse-gets-first-vaccine-shot-in-new-york-city11607958012?mod=article_inline (2020).

20. Luthra, S. Pregnant women haven't been included in promising COVID-19 vaccine trials. USA Today https://www.usatoday.com/ story/news/nation/2020/11/23/pregnant-women-excluded-pfizermoderna-covid-19-vaccine-trials/6352679002/ (2020).

21. Lovelace Jr., B. Doctors say CDC should warn people the side effects from Covid vaccine shots won't be 'a walk in the park'. CNBC https://www.cnbc.com/2020/11/23/covid-vaccine-cdc-should-warnpeople-the-side-effects-from-shots-wont-be-walk-in-the-park-.html (2020).

22. Kaiser, J. Temperature concerns could slow the rollout of new coronavirus vaccine. Science https://www.sciencemag.org/ news/2020/11/temperature-concerns-could-slow-rollout-newcoronavirus-vaccines (2020). 